\title{
microRNA-22 attenuates myocardial ischemia-reperfusion injury via an anti-inflammatory mechanism in rats
}

\author{
JIAN YANG $^{1 *}$, ZHIXING FAN $^{1 *}$, JUN YANG $^{1}$, JIAWANG DING $^{1}$, CHAOJUN YANG $^{1}$ and LIHUA CHEN ${ }^{2}$ \\ ${ }^{1}$ Department of Cardiology, Institute of Cardiovascular Diseases, The First College of Clinical Medical Sciences; \\ ${ }^{2}$ Department of Optometry and Ophthalmology, Yichang Central People's Hospital, \\ China Three Gorges University, Yichang, Hubei 443000, P.R. China
}

Received May 23, 2015; Accepted July 15, 2016

DOI: $10.3892 /$ etm.2016.3777

\begin{abstract}
Previous studies have reported that microRNA-22 (miR-22) may be implicated in ischemia-reperfusion (I/R)-induced myocardial injury. Our previously published data also demonstrated that miR-22 may protect against myocardial I/R injury via anti-apoptosis in rats by targeting cAMP response element-binding protein binding protein (CBP). However, the specific function of miR-22 in myocardial I/R injury is far from fully elucidated. The present study was designed to investigate another cardioprotective signaling mechanism of miR-22 in myocardial I/R injury. A total of 40 adult male Sprague-Dawley rats were randomly divided into four equal groups $(\mathrm{n}=10)$ : Sham, myocardial $\mathrm{I} / \mathrm{R}$, myocardial I/R with adenovirus expressing scramble miRNA (Ad-Scramble) and myocardial I/R with adenovirus expressing miR-22 (Ad-miR-22) groups. Besides the Sham operation group, the remaining three groups were artificially afflicted with coronary occlusion for $30 \mathrm{~min}$ and subsequently reperfused for $4 \mathrm{~h}$. A light microscope was used to observe structural changes in the myocardium; reverse transcription polymerase chain reaction was used to measure the miR-22 mRNA expression level; the myocardial infarct size was analyzed by the Evans Blue/triphenyltetrazolium chloride double-staining; and p38 mitogen-activated protein kinase (MAPK), CBP, c-Jun-activator protein (AP)-1 and phospho (p)-c-Jun-AP-1 expression protein levels were detected by a western blot. Furthermore, ELISA was used to measure the levels of TNF- $\alpha$ and IL- 6 in the myocardium. The results demonstrated that adenovirus-mediated miR-22 overexpression markedly
\end{abstract}

Correspondence to: Dr Jian Yang, Department of Cardiology, Institute of Cardiovascular Diseases, The First College of Clinical Medical Sciences, China Three Gorges University, 183 Yiling Road, Yichang, Hubei 443000, P.R. China

E-mail: yichangyangjian@163.com

${ }^{*}$ Contributed equally

Key words: microRNAs, reperfusion injury, mitogen-activated protein kinase, cAMP response element-binding protein binding protein, activator protein 1 , inflammation reduced p38 MAPK, CBP, c-Jun-AP-1, p-c-Jun-AP-1 expression levels concomitant with an improvement in myocardial injury, including smaller infarct size, reduced release of creatine kinase, lactate dehydrogenase and proinflammation mediators (tumor necrosis factor- $\alpha$ and interleukin-6). These findings suggest that miR-22 has a protective effect on myocardial I/R injury. This protection mechanism, at least in part, is due to its anti-inflammatory function via the suppression of the p38 MAPK/CBP/c-Jun-AP-1 signaling pathway.

\section{Introduction}

It is widely recognized that re-establishing the blood flow to ischemic myocardial tissue has become the most viable therapeutic approach for the treatment of ischemic heart disease (1). However, subsequent ischemia/reperfusion (I/R) injury may reduce the therapeutic benefit (2). Indeed, a wide range of pathological processes contribute to myocardial I/R injury (3), and it has been widely accepted that inflammation is important in myocardial I/R injury (4). There is comprehensive experimental and clinical evidence that anti-inflammatory actions attenuate $\mathrm{I} / \mathrm{R}$ injury $(5,6)$. It is therefore necessary that an effective therapeutic target against inflammation is identified to attenuate I/R injury.

microRNAs (miRNAs) are a class of high abundance, evolutionarily conserved, non-coding, small single-stranded RNAs that negatively regulate gene expression by binding to the 3 ' untranslated region of target mRNAs (7). miRNAs regulate genes involved in a diverse range of biological processes by this mechanism, including development, differentiation, inflammation, stress responses, angiogenesis, adhesion, proliferation and apoptosis $(8,9)$. In addition, miRNAs are also recognized as critical regulators of cardiovascular diseases (10). A previous study revealed that miRNAs also have an important role in myocardial I/R injury (11). Recently, one specific miRNA, miR-22 was observed to have decreased in rat hearts after I/R (12). These data indicate that miR-22 may also be involved in the pathological progression of myocardial I/R injury. Furthermore, our previously published data also demonstrated that adenovirus-mediated miR-22 overexpression may protect against myocardial I/R injury via anti-apoptosis in rats by targeting cAMP response element-binding protein binding protein (CBP) (12). However, 
the specific function of miR-22 in myocardial I/R injury is far from fully elucidated.

p38-mitogen-activated protein kinase (p38-MAPK) and CBP are general transcriptional co-activators, which are capable of phosphorylating various sequence-specific transcription factors, including activator protein (AP)-1 and p53, in order to regulate their downstream gene expression (13-15). AP-1 is a regulator of cytokine expression and a significant modulator in inflammatory diseases, including rheumatoid arthritis, psoriasis, psoriatic arthritis and myocardial I/R injury (16). c-Jun is considered to be a dominant component of the AP-1 complex (c-Jun-AP-1) (17). p38 MAPK and CBP have been identified as potential miR-22 targets using Target-Scan bioinformatics software. A previous unpublished study performed in our laboratory also identified that the levels of p38 MAPK, CBP and c-Jun-AP-1 were upregulated in rat hearts following I/R. However, it is remains unknown whether miR-22 is able to protect against myocardial $\mathrm{I} / \mathrm{R}$ injury through anti-inflammation in rats via the suppression of 338 MAPK, CBP and c-Jun-AP-1.

In the present study, adenoviral overexpression of miR-22 was used to investigate another cardioprotective signaling mechanism of miR-22 in myocardial I/R injury. The data demonstrated that miR-22 was able to efficiently attenuate I/R injury by regulating p38 MAPK, CBP and c-Jun-AP-1 expression and inhibiting inflammation.

\section{Materials and methods}

Animals. A total of 40 adult male Sprague-Dawley rats (weight, 220-250 g) were purchased from China Three Gorges University (CTGU; Yichang, China). Rats received a standard diet and free access to water, and were maintained at $23 \pm 3^{\circ} \mathrm{C}$ with a $12 \mathrm{~h}$ light/dark cycle. The procedures for experiments and animal care were approved by the Animal Care and Use Committee of CTGU, and conformed to the Guide for the Care and Use of Laboratory Animals outlined by the National Institutes of Health (NIH publication no. 80-23).

Construction of miR-22 expression adenoviral vector. Rno-miR-22 precursor DNA (MI0000851) was synthesized by Genechem, Co., Ltd., (Shanghai, China). The adenovirus expressing miR-22 (Ad-miR-22) or control adenovirus expressing scramble miRNA (Ad-Scramble) were generated using the AdMax system (Microbix Biosystems, Ontario, Canada) according to the manufacturer's instructions. These resulting adenoviruses were subsequently packaged and amplified in HEK293 cells (Sunbio, Inc., Shanghai, China), and purified using cesium chloride banding (12). Viral titer was routinely concentrated to $\sim 1 \times 10^{9}$ plaque-forming unit (PFU) as determined by the plaque assay.

In vivo gene transfer and establishment of a myocardial I/R model. Rats were subjected to adenovirus-mediated gene transfer and a subsequent myocardial I/R surgical procedure. The myocardial ischemia reperfusion model was performed as explained in a previous study (12). Briefly, rats were anesthetized with pentobarbital $(30 \mathrm{mg} / \mathrm{kg})$ via intraperitoneal injection ventilated with oxygen using a small animal breathing machine. After gently opening the chest through the fourth and fifth ribs and safely exposing the heart, a $100 \mu \mathrm{l}$ solution of Ad-miR-22 $\left(1 \times 10^{9} \mathrm{PFU}\right)$, Ad-Scramble $\left(1 \times 10^{9} \mathrm{PFU}\right)$ or saline was respectively injected into six separate sites of the left ventricular anterior wall using a 26-gauge needle. Following the adenovirus or saline delivery, the chest was closed and the rat was allowed to recover from anesthesia. Four days after the rats were re-anesthetized with pentobarbital $(30 \mathrm{mg} / \mathrm{kg})$ and the chest was re-opened allowing $100 \%$ oxygen ventilation via a small animal breathing machine. The thorax was reopened through the original intercostal space and the left anterior descending coronary artery (LAD) was identified. LAD was ligated using a 6-0 silk suture. Additionally, a medical latex tube (socket inner diameter, $1.5 \mathrm{~mm}$ ) was placed between the ligature and the LAD. Myocardial ischemia was induced by tightening the ligature around the latex tube. Successfully surgical myocardial ischemia was detected on the basis of S-T segment elevation on an electrocardiogram. After $30 \mathrm{~min}$, the suture was withdrawn to restore normal circulation for a $4 \mathrm{~h}$ period of reperfusion. Following $4 \mathrm{~h}$ of reperfusion, the hearts of the rats and blood samples were harvested for further investigation. Sham-operated rats underwent similar procedures without the occlusion of LAD and myocardial ischemic reperfusion.

Biochemical studies. Blood serum samples were collected to measure the expression levels of two specific marker enzymes, CK (03010702011) and LDH (03010703011), using commercial kits (Beijing Kemeidongya Biotechnology Ltd., Beijing, China), according to the manufacturer's instructions. The results were presented as U/1.

Detection of myocardial infarct area (IA)/area at risk (AAR). Evans blue/triphenyltetrazolium chloride (TTC) double-staining was performed to determine the IA of the myocardium as previously described (12). In brief, following $4 \mathrm{~h}$ reperfusion, LAD was immediately re-occluded and $1 \mathrm{ml} 2.0 \%$ Evans blue (Sigma-Aldrich, St. Louis, MO, USA) was intravenously administered to discriminate between the viable non-ischemic area and the zone at risk. Stained hearts were quickly removed, frozen and sliced to yield five sections ( 2-mm thick), which were subsequently incubated in $1.5 \%$ TTC (Sigma-Aldrich) for $15 \mathrm{~min}$ at $37^{\circ} \mathrm{C}$. The viable myocardium at risk of ischemic was stained red with TTC, whereas the IA without staining was pale white. Therefore, the AAR (red plus white) and IA (white) from each slice were delineated and assessed using Image-Pro Plus 5.0 software (Media Cybernetic, Rockville, MD, USA). The percentage of IA/AAR was calculated.

Histological examination. Formalin-fixed, paraffin-embedded sections of myocardial tissues were stained with hematoxylin and eosin and examined under a light microscope (magnification, $\mathrm{x} 400$ ).

Total RNA extraction and reverse transcription quantitative polymerase chain reaction ( $R T-q P C R)$. Total RNA from the cardiac muscle samples was extracted using $1 \mathrm{ml}$ TRIzol reagent (Thermo Fisher Scientific, Inc., Waltham, MA, USA) per $100 \mathrm{mg}$ of tissue using a glass homogenizer. For miRNA detection, $4.0 \mu \mathrm{g}$ RNA was reverse transcribed using a commercial cDNA synthesis kit (Fermentas; Thermo Fisher Scientific, Inc.) at $42^{\circ} \mathrm{C}$ for $1 \mathrm{~h}$. A mirVana RT-qPCR miRNA detection kit (Thermo Fisher Scientific, Inc.) was used to measure miR-22 expression 
levels. Amplification and detection via qPCR were performed in a total reaction volume of $20 \mu \mathrm{l}$ consisting of SYBR Premix Ex Taq II $(10 \mu \mathrm{l}), 50 \mathrm{X}$ ROX Reference Dye II $(0.4 \mu \mathrm{l})$, forward primer $(0.8 \mu \mathrm{l})$, reverse primer $(0.8 \mu \mathrm{l})$, DNA-template $(2 \mu \mathrm{l})$ and nuclease-free water $(6 \mu \mathrm{l})$, using an ABI Prism 7500 system (Thermo Fisher Scientific, Inc.), and U6 was used as an internal control. The relative level of miR-22 was calculated based on the $2^{-\triangle \Delta C \mathrm{q}}$ method (18). qPCR was run as follows: $50^{\circ} \mathrm{C}$ for $2 \mathrm{~min}$, $95^{\circ} \mathrm{C}$ for $10 \mathrm{~min}$, and 40 cycles of $95^{\circ} \mathrm{C}$ for $30 \mathrm{sec}$ and $60^{\circ} \mathrm{C}$ for $30 \mathrm{sec}$. The following sequence-specific primers were used to amplify the gene products: miR-22, forward, 5'-TGCGCA GTTCTTCAGTGGCAAG-3' and reverse, 5'-CCAGTGCAG GGTCCGAGGTATT-3'; and U6, forward, 5'-CGCTTCGGC AGCACATATAC-3' and reverse, 5'-AAATATGGAACGCTT CACGA-3'. Samples were examined in triplicate.

Western blot analysis. To determine the protein levels of p38 MAPK, CBP, c-Jun-AP-1, phospho (p)-c-Jun-AP-1 and GAPDH in the myocardial tissue, protein was extracted from the AAR of the heart and western blot analysis was performed as previously described (12). Briefly, the extracted proteins $(50 \mu \mathrm{g})$ were separated by $10 \%$ sodium dodecyl sulfate-polyacrylamide gel and transferred onto nitrocellulose membranes. Non-specific binding was blocked with $5 \%$ non-fat dry milk for $2 \mathrm{~h}$ at room temperature. The membrane was subsequently rinsed and incubated with anti-p38 MAPK (sc-535; 1:500), anti-CBP (sc-632; 1:500), anti-c-Jun-AP-1 (sc-44; 1:1,000) and anti-p-c-Jun-AP-1 (sc-101721; 1:1,000) primary antibodies overnight at $4^{\circ} \mathrm{C}$. Following washing three times with TBST, the membranes were incubated with peroxidase-conjugated secondary antibodies (1:50,000; BA1054; Boster Biological Technology, Ltd., Wuhan, China) for $2 \mathrm{~h}$ at room temperature. Bands were visualized using an enhanced chemiluminescence detection kit (Pierce Biotechnology, Inc., Rockford, IL, USA). The expression level of GAPDH served as a loading control and was used to normalize the densities of the different samples. ImageJ 6.0 software (National Institutes of Health, Bethesda, MA, USA) was used to quantify the optical density of each band.

ELISA. The ELISA method was used to determine the TNF- $\alpha$ and IL-6 levels in cardiac muscle samples according to the manufacturer's instructions. TNF- $\alpha$ (F16960) and IL-6 (F15870) ELISA kits from Xitang Company (Shanghai, China) were used.

Statistical analysis. All the values were presented as the mean \pm standard error of the mean. Differences between groups were analyzed for significance using one-way analysis of variance and Student-Newman-Keuls- $q$ test using SPSS software (version 14.0; SPSS Inc., Chicago, IL, USA). P<0.05 was used to indicate a statistically significant difference.

\section{Results}

Myocardial I/R induces the downregulation of $m i R-22$ expression levels. Following 4-h reperfusion the levels of miR-22 expressed by the I/R myocardium were significantly reduced in comparison to the non-ischemic sham control (I/R vs. sham group; $\mathrm{P}<0.05$; Fig. 1). Following transfection to induce adenoviral overexpression of

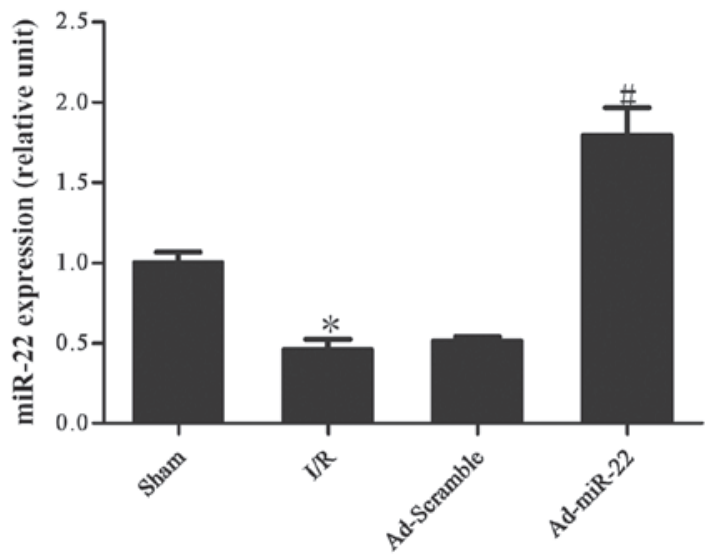

Figure 1. miR-22 expression was reduced after myocardial I/R injury, while delivery of Ad-miR-22 into the myocardium improved the expression of miR-22. Data are expressed as the mean \pm standard error of the mean $(n=6) .{ }^{*} \mathrm{P}<0.05$ vs. the sham group and ${ }^{\#} \mathrm{P}<0.05$ vs. the $\mathrm{I} / \mathrm{R}$ group. $\mathrm{I} / \mathrm{R}$, ischemia-reperfusion; Ad, adenovirus.

miR-22 into the I/R myocardium for 4 days, miR-22 expression was significantly increased (Ad-miR-22 vs. I/R group; $\mathrm{P}<0.05)$. However, Ad-Scramble had no apparent effect on the expression level of miR-22 compared with the I/R group (Ad-Scramble vs. I/R group; P>0.05).

miR-22 reduces serum marker enzyme levels. The activities of $\mathrm{CK}$ and $\mathrm{LDH}$ in the serum were used to monitor the damage to the myocardium. Serum CK and LDH activity significantly increased after $4 \mathrm{~h}$ of reperfusion in the I/R group compared with the sham group (I/R vs. sham group; $\mathrm{P}<0.05$; Fig. 2). However, after Ad-miR-22 transfection, the $\mathrm{CK}$ and LDH levels in the Ad-miR-22 group significantly decreased in comparison to the I/R group (Ad-miR-22 vs. I/R group; $\mathrm{P}<0.05)$. Ad-Scramble did not affect $\mathrm{I} / \mathrm{R}$-induced increased leakage of CK and LDH from the myocardium (Ad-Scramble group vs. I/R group; P>0.05).

Upregulation of miR-22 decreases infarct size. To quantitatively analyze the damage and potential prognosis after reperfusion, IA/AAR was introduced as described previously. Infarct size was measured as $51.4 \pm 2.6 \%$ of the IA/AAR in the I/R group. Furthermore, overexpression of miR-22 using a recombinant adenoviral vector exerted cardioprotective effects on the development of myocardial I/R injury by significantly reducing IR/AAR (Ad-miR-22 vs. I/R group; $\mathrm{P}<0.05$; Fig. 3). Transfection of Ad-Scramble had no significant effect on the damage and prognosis after myocardial $\mathrm{I} / \mathrm{R}$ in comparison with the I/R group (Ad-Scramble vs. $\mathrm{I} / \mathrm{R}$ group; $\mathrm{P}>0.05$ ). This demonstrates that overexpression of miR-22 may be used as a cardioprotective agent against myocardial I/R injury.

Light microscopy evaluation. Myocardial fibers of the Sham group were arranged regularly without any apparent degeneration or necrosis. However, disorganized myocardial fibers, edema, and ruptured and lysed cells were observed in the I/R group. Furthermore, delivery of miR-22 partially rescued myocardium injury and inflammatory cell infiltration. In 


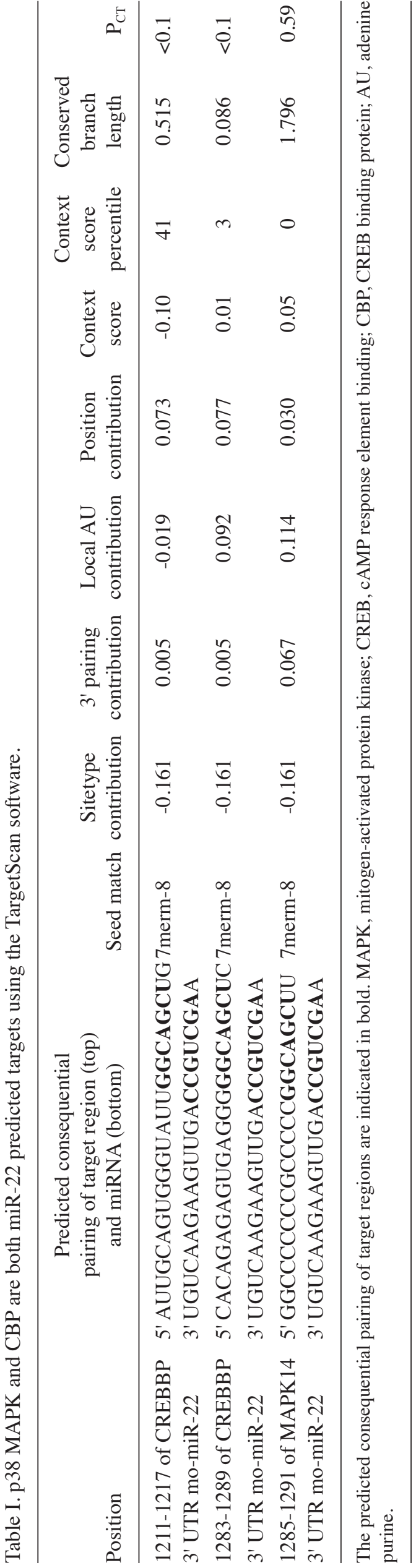

A

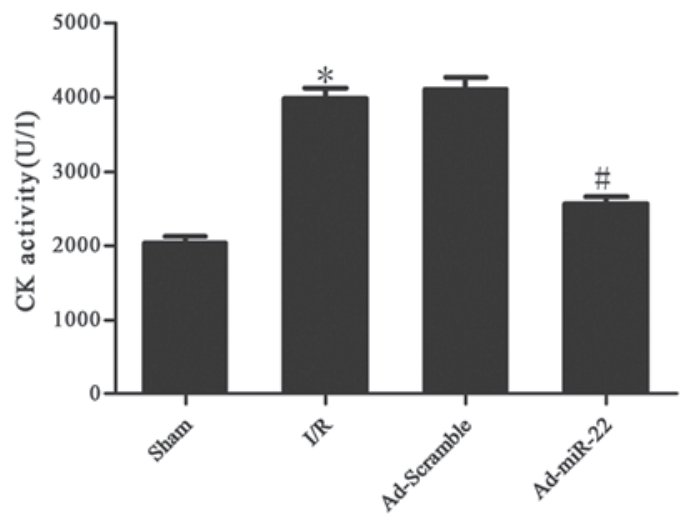

B

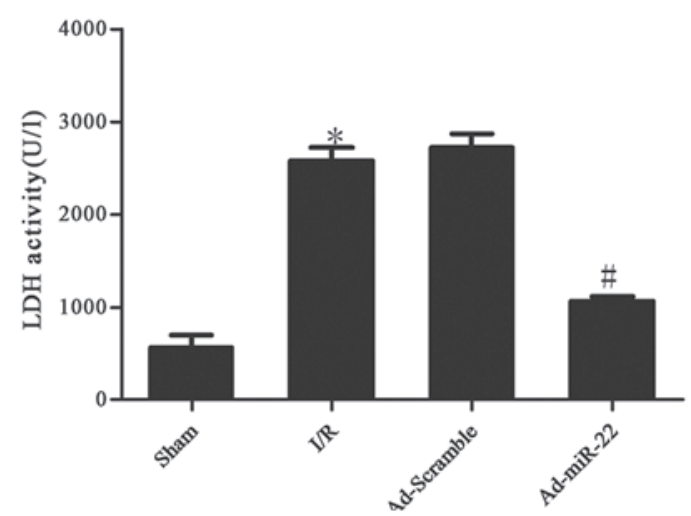

Figure 2. (A) CK and (B) LDH activity levels were reduced following delivery of Ad-miR-22. Data are expressed as the mean \pm standard error of the mean $(n=6)$. ${ }^{*} \mathrm{P}<0.05$ vs. the sham group and ${ }^{\#} \mathrm{P}<0.05$ vs. the I/R group. $\mathrm{CK}$, creatine kinase; $\mathrm{LDH}$, lactate dehydrogenase; I/R, ischemia-reperfusion; Ad, adenovirus.

$\mathbf{A}$

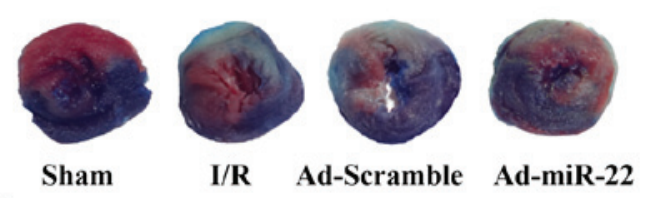

B

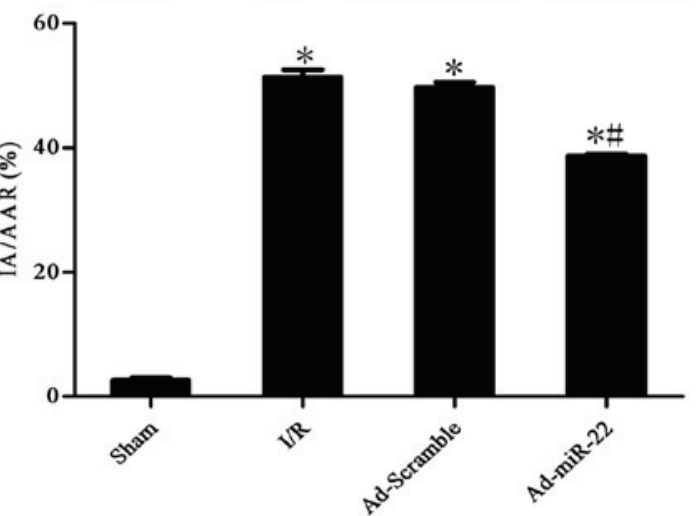

Figure 3. Upregulation of miR-22 decreased infarct size following I/R injury. (A) Representative pictures of Evans and triphenyltetrazolium chloride staining of the areas of myocardial infarction. (B) Infarct size was presented as a percentage of the area at risk (IA/AAR). Data are expressed as the mean \pm standard error of the mean $(\mathrm{n}=3)$. ${ }^{*} \mathrm{P}<0.05$ vs. the sham group and ${ }^{\text {"}} \mathrm{P}<0.05$ vs. the $\mathrm{I} / \mathrm{R}$ group. I/R, ischemia-reperfusion; IA, myocardial infarct size; AAR, area at risk; Ad, adenovirus.

addition, transfection with Ad-Scramble had no notable effect on the morphological changes, compared with the I/R group (Fig. 4). 

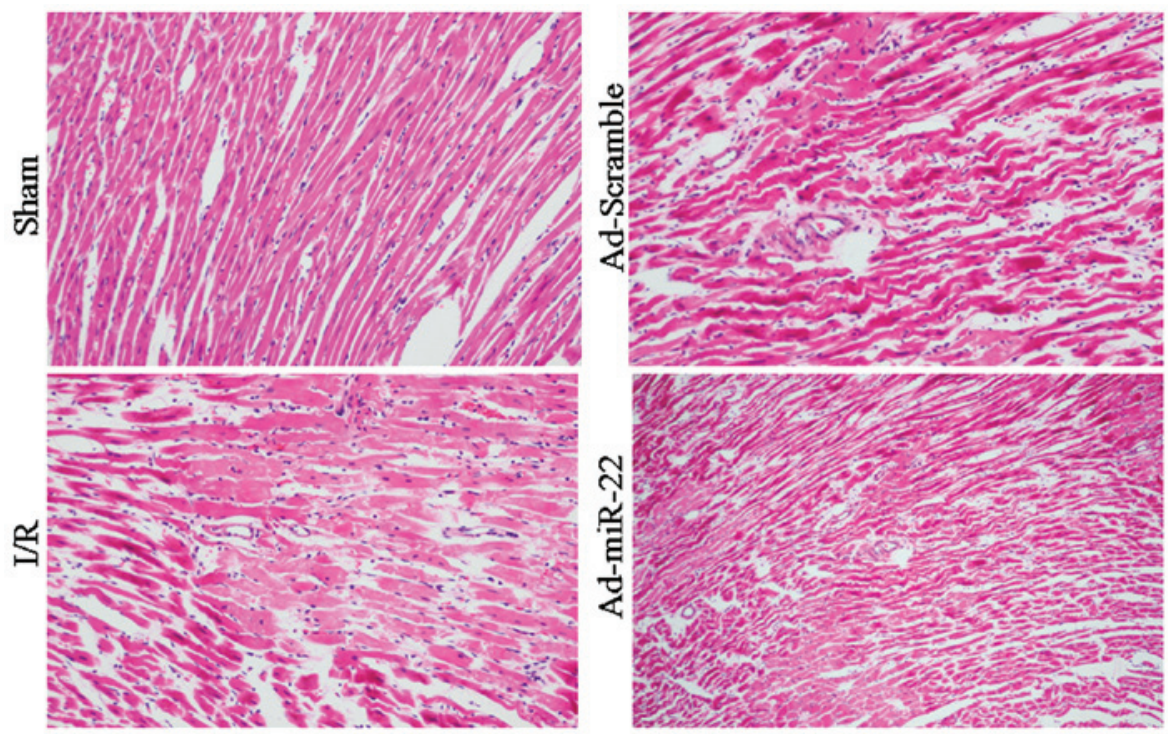

Figure 4. Histopathological changes in the myocardium of the different groups (magnification, x400). In the Sham group, myocardial fibers were arranged regularly. In the I/R group, the myocardium was swollen, ruptured and inflammatory cell infiltration was also observed. In the Ad-Scramble group, the morphology exhibited apparent degeneration and necrosis compared with the I/R group. In the Ad-miR-22 group, well-arranged cardiac cells and a minimal amount of inflammatory cell infiltration were observed. I/R, ischemia-reperfusion; Ad, adenovirus.

A

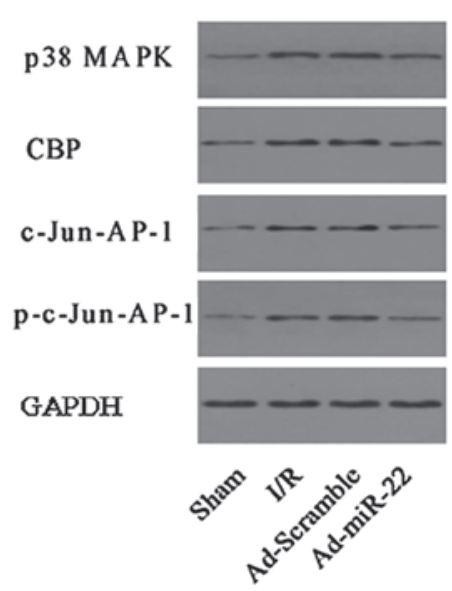

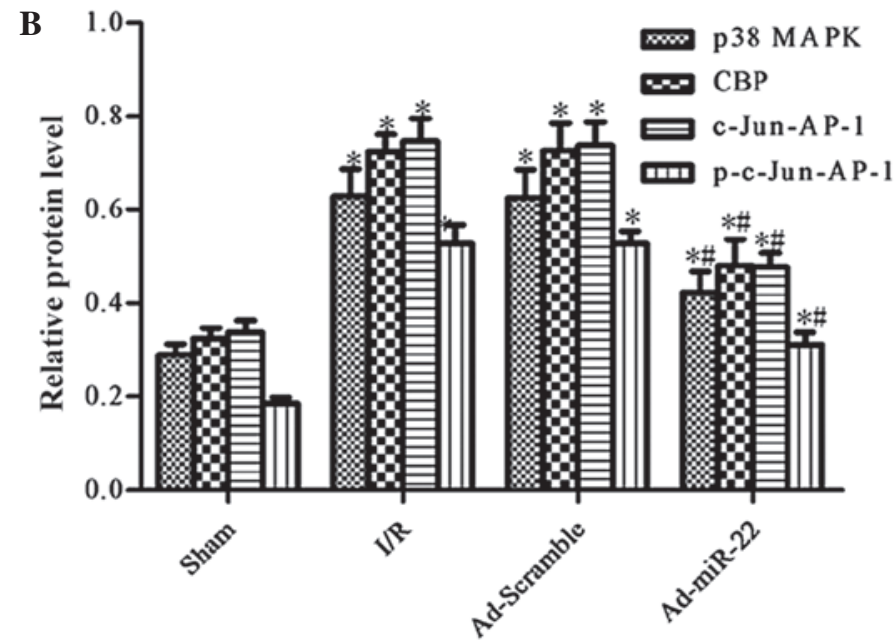

Figure 5. Upregulation of miR-22 suppressed the expression of p38 MAPK/CBP/c-Jun-AP-1 signaling pathway. (A) Original representative western blots of the proteins obtained from myocardial tissue. (B) Relative p38 MAPK, CBP, c-Jun-AP-1, p-c-Jun-AP-1 protein levels in the four groups. GAPDH was used as the internal control. Data are presented as the mean \pm standard error of the mean $(n=6) .{ }^{*} \mathrm{P}<0.05$ vs. the Sham group; ${ }^{*} \mathrm{P}<0.05$ vs. the I/R group. MAPK, mitogen-activated protein kinase; CREB, cAMP response element binding; CBP, CREB binding protein; GAPDH, glyceraldehyde 3-phosphate dehydrogenase; AP-1, activator protein-1; Ad, adenovirus.

Upregulation of miR-22 suppresses the expression of the $p 38 \mathrm{MAPK} / \mathrm{CBP} / \mathrm{c}-\mathrm{Jun}-\mathrm{AP}-1$ signaling pathway. p38 MAPK and CBP were demonstrated to be predicted target genes of miR-22 by TargetScan (12) (genes.mit.edu/tscan/targetscan2003.html; Table I). c-Jun is a dominant component of the c-Jun-AP-1 transcription factor, and p-c-Jun-AP-1 is the active form of c-Jun-AP-1 (17). Compared to the Sham group, the aforementioned protein levels were both significantly upregulated in the I/R injury group (I/R vs. sham group; $\mathrm{P}<0.05$; Fig. 5). Furthermore, delivery of miR-22 into the myocardium significantly reduced the levels of p38 MAPK, CBP, c-Jun-AP-1 and p-c-Jun-AP-1 by $33.12,32.90$ and $38.50 \%$, respectively (Ad-miR-22 vs. I/R group; $\mathrm{P}<0.05$ ). In addition, adenoviral transfection of Ad-Scramble had no significant effect on the proteins mentioned above compared with the I/R group (Ad-Scramble vs. I/R group; $\mathrm{P}>0.05$ ). Therefore, overexpression of miR-22 may specifically suppress the expression of the p38 MAPK/CBP/c-Jun-AP-1 signaling pathway.

miR-22 reduces the levels of TNF- $\alpha$ and IL-6. Myocardial $\mathrm{I} / \mathrm{R}$ induced a significant increase in the concentrations of TNF- $\alpha(37.99 \pm 0.13$ vs. $19.43 \pm 0.69 \mathrm{pg} / \mathrm{mg} ; \mathrm{P}<0.05)$ and IL-6 $(49.66 \pm 0.85$ vs. $26.45 \pm 0.60 \mathrm{pg} / \mathrm{mg} ; \mathrm{P}<0.05)$ in the $\mathrm{I} / \mathrm{R}$ group in comparison with the sham group. Myocardial delivery of miR-22 significantly inhibited TNF- $\alpha(30.15 \pm 0.21$ vs. $37.99 \pm 0.13 \mathrm{pg} / \mathrm{mg} ; \mathrm{P}<0.05)$ and IL-6 (39.50 \pm 0.55 vs. $49.66 \pm 0.85$ pg/mg; $\mathrm{P}<0.05)$ expression 
compared with the I/R group. However, adenoviral transfection of Ad-Scramble did not have a significant effect on the two aforementioned cytokines in comparison with the I/R group (Ad-Scramble vs. I/R group; $\mathrm{P}>0.05$ ). These data suggest that miR-22 could inhibit the production of inflammation cytokines (Table II and Fig. 6).

\section{Discussion}

It has previously been demonstrated that inflammation pathways have a significant role in the pathophysiological process of myocardial I/R injury (19). Experimental and clinical evidence has indicated that anti-inflammatory actions may attenuate I/R injury $(5,6)$. Utilizing adenovirus-associated vectors, the present study demonstrated that selective overexpression of miR-22 induced promisingly cardioprotective properties as well as an anti-inflammatory role in ameliorating myocardial $\mathrm{I} / \mathrm{R}$ injury in vivo. After increasing the levels of miR-22, the infarct size and disordered morphology and myocardial enzyme levels were reduced. Meanwhile, concomitant p38 MAPK, CBP, c-Jun-AP-1, p-c-Jun-AP-1 suppression and inflammation cytokine (TNF- $\alpha$ and IL-6) reduction occurred. These major findings demonstrated that the cardioprotective effect of miR-22 against inflammation is, at least partly, functionally attributed to its suppression of the p38 MAPK/CBP/c-Jun-AP-1 signaling pathway.

A number of miRNAs have been demonstrated to be involved in myocardial I/R injury and miR-22 was only one of these reported to regulate $I / R$ injury $(12,20)$. Our previous study also demonstrated that adenovirus-mediated miR-22 overexpression protected against myocardial I/R injury through an anti-apoptosis mechanism in rats by targeting CBP (12). However, the molecular mechanisms involved in the cardioprotective effect of miR-22 are complicated and are far from fully understood.

As a cardiac-enriched miRNA, miR-22 has various targets in cardiomyocytes that were identified using TargetScan (12). p38 MAPK and CBP are both targets (Table I). In the present study, p38 MAPK and CBP were upregulated in the I/R group; however, both were suppressed following the delivery of miR-22. The transcription factor, AP-1, had a similar variation tendency with p38 MAPK and CBP in the four groups. This indicated that AP-1 was also suppressed after p38 MAPK and CBP was suppressed by miR-22.

p38 MAPK, which is one of the most functional members of the MAPK family, has a key role in the progression of I/R injury and is also involved in the activation of the transcription factor, AP-1 $(21,22)$. The CBP gene is widely expressed and is important in the cardiovascular system as it interacts with a variety of diverse transcriptional factors, including AP-1 and p53 (12,23-25). Our previously published data also demonstrated that CBP may be important in myocardial I/R injury by influencing p53 (12). In summary, p38 MAPK and CBP may both activate AP-1. Furthermore, p38 MAPK may not only activate AP-1 directly but may also activate CBP, and as a consequence activate AP-1 (26). AP-1 is a regulator of cytokine expression and is an important modulator in inflammatory diseases, including rheumatoid arthritis, psoriasis, psoriatic arthritis and myocardial I/R injury (16). c-Jun is considered to be a dominant component of the c-Jun-AP-1 transcription
Table II. Myocardial TNF- $\alpha$ and IL-6 expression after $4 \mathrm{~h}$ reperfusion in the four groups.

\begin{tabular}{lcl}
\hline Group & TNF- $\alpha(\mathrm{pg} / \mathrm{mg})$ & IL-6 $(\mathrm{pg} / \mathrm{mg})$ \\
\hline Sham & $19.43 \pm 0.69$ & $26.45 \pm 0.60$ \\
I/R & $37.99 \pm 0.13^{\mathrm{a}}$ & $49.66 \pm 0.85^{\mathrm{a}}$ \\
Ad-Scramble & $38.65 \pm 0.56^{\mathrm{a}}$ & $49.61 \pm 0.51^{\mathrm{a}}$ \\
Ad-miR-22 & $30.15 \pm 0.21^{\mathrm{a}, \mathrm{b}}$ & $39.50 \pm 0.55^{\mathrm{a}, \mathrm{b}}$ \\
\hline
\end{tabular}

Mean \pm standard error of the mean, $n=6,{ }^{a} \mathrm{P}<0.05$ vs. Sham group and ${ }^{\mathrm{b}} \mathrm{P}<0.05$ vs. the I/R group. TNF- $\alpha$, tumour necrosis factor- $\alpha$; IL- 6 , interleukin-6; I/R, ischemia-reperfusion; Ad, adenovirus.
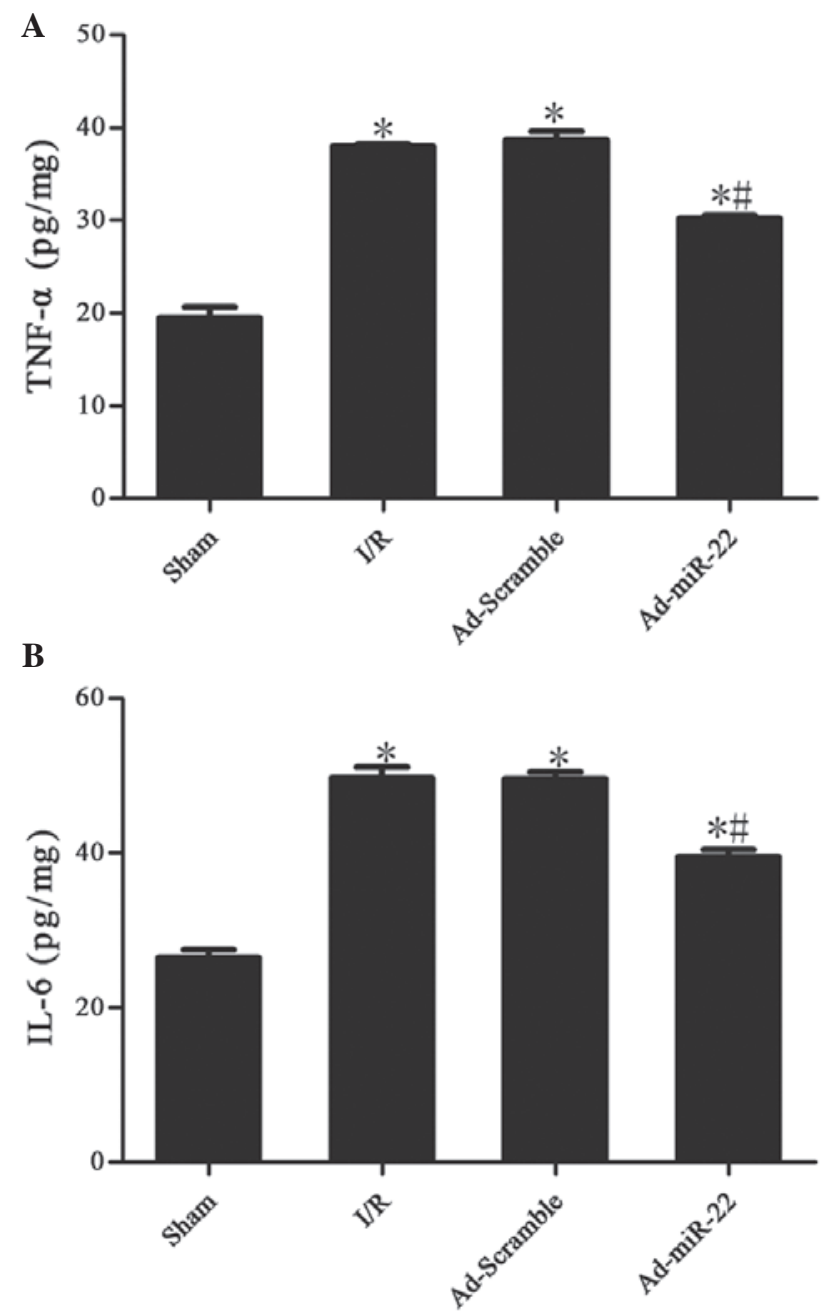

Figure 6. Upregulation of miR-22 reduced the expression levels of (A) TNF- $\alpha$ and (B) IL-6. Data are expressed as the mean \pm standard error of the mean $(n=6) .{ }^{*} \mathrm{P}<0.05$ vs. the sham group; ${ }^{\#} \mathrm{P}<0.05$ vs. the $\mathrm{I} / \mathrm{R}$ group. TNF- $\alpha$, tumor necrosis factor- $\alpha$; IL-6, interleukin 6 ; I/R, ischemia-reperfusion; Ad, adenovirus.

factor complex and the phosphorylation of this complex is the most important regulator of c-Jun-AP-1, which suggests transcriptional activity (17,27). Hence, suppressing c-Jun-AP-1 activity reduces I/R induced myocardial injury by suppressing the inflammation caused by p-c-Jun-AP-1. The present study revealed that the production of inflammatory cytokines (TNF- $\alpha$ 
and IL-6) decreased with the suppression of p38 MAPK, CBP, c-Jun-AP-1 and p-c-Jun-AP-1 compared with the Sham group and after the overexpression of miR-22. These results indicate that anti-inflammation action may be an additional cardioprotective effect induced by miR-22 in myocardial I/R injury, and the mechanism is associated with the p38 MAPK/CBP/c-Jun-AP-1 signaling pathway.

In conclusion, the results of the present study suggests that miR-22 is capable of diminishing myocardial I/R injury induced by inflammation in rat models by directly targeting p38 MAPK and CBP. Overexpression of miR-22 leads to a significant repression of the p38 MAPK/CBP/c-Jun-AP-1 signaling pathway and results in the amelioration of inflammation. These findings suggest that overexpression of miR-22 may be a desirable therapeutic approach for the treatment of myocardial I/R injury.

\section{Acknowledgements}

The present study was supported by the National Natural Science Foundation of China (grant nos. 81170133, 81200088 and 81470387), the Master's Degree Paper Pew Foundation of China Three Gorges University (grant no. 2015PY052) and Hubei Province's Outstanding Medical Academic Leader program of China (grant no. 201304).

\section{References}

1. Ndrepepa G: Improving myocardial injury, infarct size, and myocardial salvage in the era of primary PCI for STEMI. Coron Artery Dis 26: 341-355, 2015.

2. Kloner RA: Does reperfusion injury exist in humans? J Am Coll Cardiol 21: 537-545, 1993.

3. Eltzschig HK and Eckle T: Ischemia and reperfusion - from mechanism to translation. Nat Med 17: 1391-1401, 2011.

4. Hu H, Zhai C, Qian G, Gu A, Liu J, Ying F, Xu W, Jin D, Wang H, $\mathrm{Hu} \mathrm{H}$, Zhang Y and Tang G: Protective effects of tanshinone IIA on myocardial ischemia reperfusion injury by reducing oxidative stress, HMGB1 expression and inflammatory reaction. Pharm Biol 53: 1752-1758, 2015.

5. Du X, Hu X and Wei J: Anti-inflammatory effect of exendin-4 postconditioning during myocardial ischemia and reperfusion. Mol Biol Rep 41: 3853-3857, 2014.

6. Doddakula KK, Neary PM, Wang JH, Sookhai S, O'Donnell A, Aherne T, Bouchier-Hayes DJ and Redmond HP: The antiendotoxin agent taurolidine potentially reduces ischemia/reperfusion injury through its metabolite taurine. Surgery 148: 567-572, 2010.

7. Fan ZX and Yang J: Microribonucleic acids and vascular restenosis. Saudi Med J 35: 796-801, 2014.

8. Suzuki HI and Miyazono K: Emerging complexity of microRNA generation cascades. J Biochem 149: 15-25, 2011.

9. Peterson SM, Thompson JA, Ufkin ML, Sathyanarayana P, Liaw L and Congdon CB: Common features of microRNA target prediction tools. Front Genet 5: 23, 2014.
10. Thum $\mathrm{T}$ and Condorelli G: Long noncoding RNAs and microRNAs in cardiovascular pathophysiology. Circ Res 116: 751-762, 2015.

11. Lorenzen JM, Batkai S and Thum T: Regulation of cardiac and renal ischemia-reperfusion injury by microRNAs. Free Radic Biol Med 64: 78-84, 2013.

12. Yang J, Chen L, Yang J, Ding J, Li S, Wu H, Zhang J, Fan Z, Dong W and Li X: MicroRNA-22 targeting CBP protects against myocardial ischemia-reperfusion injury through anti-apoptosis in rats. Mol Biol Rep 41: 555-561, 2014

13. Liu X, Shah A, Gangwani MR, Silverstein PS, Fu M and Kumar A: HIV-1 Nef induces CCL5 production in astrocytes through p38-MAPK and PI3K/Akt pathway and utilizes NF-kB, CEBP and AP-1 transcription factors. Sci Rep 4: 4450, 2014.

14. McManus KJ and Hendzel MJ: CBP, a transcriptional coactivator and acetyltransferase. Biochem Cell Biol 79: 253-266, 2001.

15. Sugden PH and Clerk A: 'Stress-responsive' mitogen-activated protein kinases (c-Jun N-terminal kinases and p38 mitogen-activated protein kinases) in the myocardium. Circ Res 83: 345-352, 1998.

16. Zenz R, Eferl R, Scheinecker C, Redlich K, Smolen J, Schonthaler HB, Kenner L, Tschachler E and Wagner EF: Activator protein 1 (Fos/Jun) functions in inflammatory bone and skin disease. Arthritis Res Ther 10: 201, 2008.

17. Karin M: The regulation of AP-1 activity by mitogen-activated protein kinases. J Biol Chem 270: 16483-16486, 1995.

18. Livak KJ and Schmittgen TD: Analysis of relative gene expression data using real-time quantitative PCR and the 2- $\Delta \Delta \mathrm{Ct}$ method. Methods 25: 402-408, 2001.

19. Ma L, Liu H, Xie Z, Yang S, Xu W, Hou J and Yu B: Ginsenoside $\mathrm{Rb} 3$ protects cardiomyocytes against ischemia-reperfusion injury via the inhibition of JNK-mediated NF- $\mathrm{NB}$ pathway: A mouse cardiomyocyte model. PLoS One 9: e103628, 2014.

20. Yang W, Shao J, Bai X and Zhang G: Expression of plasma microRNA-1/21/208a/499 in myocardial ischemic reperfusion injury. Cardiology 130: 237-241, 2015.

21. Kumphune S, Surinkaew S, Chattipakorn SC and Chattipakorn N: Inhibition of p38 MAPK activation protects cardiac mitochondria from ischemia/reperfusion injury. Pharm Biol 53: 1831-1841, 2015.

22. Surinkaew $S$, Kumphune $S$, Chattipakorn $S$ and Chattipakorn $N$ : Inhibition of p38 MAPK during ischemia, but not reperfusion, effectively attenuates fatal arrhythmia in ischemia/reperfusion heart. J Cardiovasc Pharmacol 61: 133-141, 2013.

23. Yang J, Jiang H, Chen SS, Chen J, Li WQ, Xu SK and Wang JC: Lentivirus-mediated RNAi targeting CREB binding protein attenuates neointimal formation and promotes re-endothelialization in balloon injured rat carotid artery. Cell Physiol Biochem 26: 441-448, 2010.

24. Ait-Si-AliS, Ramirez S, BarreFX, Dkhissi F, Magnaghi-Jaulin L, Girault JA, Robin P, Knibiehler M, Pritchard LL, Ducommun B, Trouche D and Harel-Bellan A: Histone acetyltransferase activity of CBP is controlled by cycle-dependent kinases and oncoprotein E1A. Nature 396: 184-186, 1998.

25. Avantaggiati ML, Ogryzko V, Gardner K, Giordano A, Levine AS and Kelly K: Recruitment of p300/CBP in p53-dependent signal pathways. Cell 89: 1175-1184, 1997.

26. Kappelmann M, Bosserhoff A and Kuphal S: AP-1/c-Jun transcription factors: Regulation and function in malignant melanoma. Eur J Cell Biol 93: 76-81, 2014.

27. Karin M and Gallagher E: From JNK to pay dirt: Jun kinases, their biochemistry, physiology and clinical importance. IUBMB Life 57: 283-295, 2005. 